\title{
Mass Calibration of the Coulter Counter Model ZM
}

\author{
M. Margarida Figueiredo, M. Graça Rasteiro, Cristina Santos, Cristina Monteiro*
}

(Received: 17 December 1990; resubmitted: 15 June 1991)

\begin{abstract}
The calibration of electrical sensing zone instruments is normally achieved by using spherical particles with a certified size. An alternative and more fundamental procedure, known as mass or self-calibration, is to use particles of the material under test.

This work concerns the mass calibration technique, in particular the equations used to calculate the mass calibration con-
\end{abstract}

stant. It is demonstrated that some of the published expressions are inconsistent. An expression particularly suitable for the Coulter Counter Model $\mathrm{ZM}$ has been derived and validated. Some experiments were also performed using irregular particles, in order to compare both calibration methods.

\section{Introduction}

The electrical sensing zone technique, sometimes called the Coulter Counter method, is widely used in particle size analysis, and often as a reference method. The Coulter Counter determines both the number and size of particles suspended in an electrically conducting liquid. The operating principles of this instrument have been extensively described in instrument instruction manuals, textbooks [1] and also in British Standard BS 3406 [2].

The calibration of these instruments is usually achieved by using spherical particles which have a known and certified size. The most popular standards are polymer latex particles which have a narrow particle size distribution. However, the instrument can also be self-calibrated, that is, calibrated with the material under analysis. This method, often known as the mass integration method, is recommended in BS 3406 as a primary calibration procedure. It is superior to latex calibration because the particle response may be affected by the shape, porosity and conductivity of the material. The procedure is therefore, more direct and traceable. This method was formerly employed for sizing BCR reference materials and polymer latex particles [3]. It is not a routine procedure, however, since it is time consuming.

The purpose of this paper is twofold : first, to compare the two calibration techniques for the Coulter Counter Model ZM, i. e. latex calibration and mass calibration, and second, to correct some misleading expressions which have been published for the mass calibration method, especially for the Coulter ZM. A corrected expression is given.

\section{Determination of the Mass Calibration Constant}

Electrical sensing zone instruments require calibration in order to assign a particle size to each of the threshold levels. The particle diameter is then calculated from the equation

$d=K_{\mathrm{d}} \sqrt[3]{T}$.

Typically, a preliminary calibration of the instrument, fitted with the appropriate orifice tube, is achieved by applying the certified latex particles and using the "half-count" method to identify the median value [4]. The calibration constant obtained in this way is automatically computed by the instrument.

The alternative calibration technique is to use the powder under analysis. In this case it is necessary to prepare a suspension of a narrow size fraction of the material and of known concentration [2]. The volume of the particles in a metered volume of suspension will be given by

$V_{\mathrm{p}}=\frac{V_{\mathrm{m}}}{V_{\mathrm{s}}} \frac{w}{\rho_{\mathrm{s}}}$

This volume can also be obtained by measuring the particle size distribution, resulting in

$V_{\mathrm{p}}=\frac{\pi}{6} \Sigma \Delta n \bar{d}^{3}$

or, substituting Eq. (1),

$V_{\mathrm{p}}=\frac{\pi}{6} K_{\mathrm{d}}^{3} \Sigma \Delta n \bar{T}$.

Comparison of Eqs. (2) and (4) gives

$K_{\mathrm{d}}=\left(\frac{6}{\pi} \frac{V_{\mathrm{m}}}{V_{\mathrm{s}}} \frac{w}{\rho_{\mathrm{s}}} \frac{1}{\Sigma \Delta n \bar{T}}\right)^{1 / 3}$.
* Prof. M. M. Figueiredo, M. G. Rasteiro, C. Santos, C. Monteiro, Departamento de Engenharia Química, Universidade de Coimbra, Largo Marquês de Pombal, 3000 Coimbra (Portugal). 
This equation is often presented in the literature in the alternative form:

$K_{\mathrm{d}}=\left(\frac{6}{\pi} \frac{V_{\mathrm{m}}}{V_{\mathrm{s}}} \frac{w}{\rho_{\mathrm{s}}} \frac{1}{\Sigma \Delta n \bar{V}}\right)^{1 / 3}$,

Here, $\bar{V}$ is defined in BS 3406 as "the arithmetic mean volume for a particular size interval", whereas in the Coulter Counter Model ZM instruction manual [3], it is defined as an "arbitrary volume". Both of these definitions, and also the notation $\bar{V}$, can be misinterpreted, since $\bar{V}$ cannot be identified with the output values of the $Z M$, nor is it mentioned how this variable can be calculated. In fact, the threshold value, $T$, for the Coulter Counter Model ZM is given by [5]

$T=\frac{T_{\mathrm{L}} \cdot A}{I \cdot \bar{G}}$.

It should be pointed out that the threshold values must be calculated for each combination of the instrument settings and therefore Eq. (5) is not the most suitable form for the Model ZM. Instead of threshold values it would be more convenient to work with volume values, which are directly available from this instrument by pressing the volume key. However, these values cannot be introduced into the equation without some mathematical manipulation.

The threshold values can be converted to volumes, or diameters, through the calibration constant. Select, for example, the latex calibration constant which is probably the last value stored in the instrument when performing a mass calibration. Then

$\Sigma \Delta n \bar{V}_{\mathrm{app}}=\frac{\pi}{6} K_{\mathrm{do}}^{3} \Sigma \Delta n \bar{T}$

Here $\bar{V}_{\text {app }}$ should be distinguished from the previous $\bar{V}$ (Eq. (6)) and is the apparent volume of the particles computed by the Model ZM when using the initial estimate of the calibration constant, $K_{\mathrm{do}}$. Substituting the value of $\Sigma \Delta n \bar{T}$ given by Eq. (8) into Eq. (5), the self-calibration constant becomes

$K_{\mathrm{d}}=K_{\mathrm{do}}\left(\frac{V_{\mathrm{ml}}}{V_{\mathrm{s}}} \frac{\mathrm{w}}{\rho_{\mathrm{s}}} \frac{1}{\Sigma \Delta n \bar{V}_{\mathrm{app}}}\right)^{1 / 3}$.

This equation yields numerical values substantially different from Eq. (6), because of the terms $\bar{V}$ and $\bar{V}_{\text {app }}$, and is the form which should be used for mass calibration.

\section{Experimental}

The test material used was a powder of irregular glass particles. For the mass integration method it is necessary to determine the sample concentration as precisely as possible. This was achieved by accurately weighing a small quantity $(15-30 \mathrm{mg})$ of a narrow sieved fraction of the glass powder and dispersing it in $300-400 \mathrm{ml}$ of electrolyte in the usual manner. Two narrow sieved fractions, obtained by wet sieving in order to remove the fines better, were used, corresponding to size classes 37-44 and 88-105 $\mu \mathrm{m}$. The density of the glass (Schott Duran 50) was measured and was equal to $2.23 \mathrm{~g} / \mathrm{cm}^{3}$. The electrolyte employed was Isoton II, which was passed through a filter of
$0.2 \mu \mathrm{m}$ pore size before use. Two different tubes were used with orifice diameters of 140 and $280 \mu \mathrm{m}$. These tubes corresponded to the two particle size fractions. The instrument was operated in the manometer mode, with coincidence correction and using a single threshold technique with 15 channels. Particular care was taken in the analysis of the coarse fraction to provide efficient stirring of the suspension and frequent flushing of the orifice tube to prevent settling of particles at the bottom of the tube. The latex calibration was performed using the "halfcount" method with a latex suspension of $19.3 \mu \mathrm{m}$ (Coulter Electronics). It was decided to use the same latex to calibrate both tubes to ensure a constant point of reference [3].

\section{Results and Discussion}

The self-calibration constant was first calculated using both Eqs. (9) and (5), being the threshold values given by Eq. (7). As expected, the results coincided. It was then decided to use Eq. (9) since it is in a more appropriate form. The summation $\Sigma \Delta n \bar{V}_{\text {app }}$ was obtained by using as initial estimate of the calibration constant $\left(K_{\mathrm{do}}\right)$ the latex calibration constant. It should be noted that any other value could be used. The results are summarized in Table 1. The values presented for the mass calibration are the mean values of at least three separate experiments. The standard deviations are given in parentheses.

Table 1: Comparison of calibration constants using the latex and the mass calibration methods.

\begin{tabular}{lllll}
\hline $\begin{array}{l}\text { Sieve fraction } \\
(\mu \mathrm{m})\end{array}$ & $\begin{array}{l}\text { Orifice tube } \\
(\mu \mathrm{m})\end{array}$ & $K_{\mathrm{d}}$ (latex) & $K_{\mathrm{d}}$ (mass) & $\begin{array}{l}\text { Difference } \\
(\%)\end{array}$ \\
\hline $37-44$ & 140 & 23.24 & $23.78(0.04)$ & 2.3 \\
$88-105$ & 280 & 49.77 & $50.85(0.60)$ & 2.2 \\
\hline
\end{tabular}

Table 2 presents the results of the particle size distributions for both fractions, listing the values of $d_{10}, d_{50}$ and $d_{90}$, obtained using the constants in Table 1 . These values are in good agreement with the sieve range.

Table 2: Results of particle size analysis using the latex and the mass calibration constants.

\begin{tabular}{lllllllll}
\hline \multirow{2}{*}{ Sicve fraction Orifice tube } & \multicolumn{3}{c}{ Latex calibration } & \multicolumn{3}{c}{ Mass calibration } \\
& & $d_{10}$ & $d_{50}$ & $d_{90}$ & $d_{10}$ & $d_{50}$ & $d_{90}$ \\
$(\mu \mathrm{m})$ & $(\mu \mathrm{m})$ & $(\mu \mathrm{m})$ & $(\mu \mathrm{m})$ & $(\mu \mathrm{m})$ & $(\mu \mathrm{m})$ & $(\mu \mathrm{m})$ & $(\mu \mathrm{m})$ \\
\hline $37-44$ & 140 & 36.72 & 42.24 & 48.10 & 37.61 & 43.20 & 49.20 \\
$88-105$ & 280 & 80.00 & 92.10 & 101.6 & 81.11 & 94.44 & 105.0 \\
\hline
\end{tabular}

Figure 1 shows the size distribution curves obtained, for one experiment, when using both calibration constants.

It is clear from the results that the mass integration values of the calibration constant agree well with the preliminary latex half-count values for both fractions. This was to be expected for non-porous particles with low aspect ratios, and has been reported by others [3]. Despite the small deviations obtained for $K_{\mathrm{d}}$ (ca. 2\%), the mass calibration method tends to yield slightly higher values of the calibration constant and the resulting particle size distribution curves are shifted correspondingly. However, this could be due to the presence of fine particles outside the measuring range of the orifice tube. 


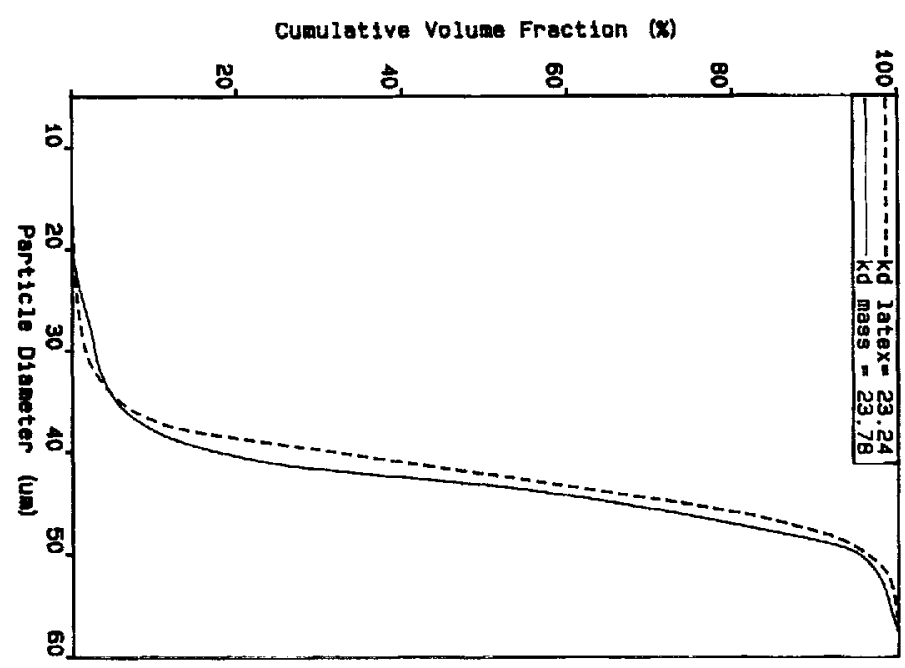

Fig. 1: Cumulative undersize distributions for a 37-44 $\mu \mathrm{m}$ sieve fraction using the latex and the mass calibration constants.

\section{Conclusions}

The difference between the particle size distributions obtained by using the latex or the mass calibration constants was found to be negligible for the material under test. Nevertheless, the mass calibration method is more fundamental and should be used to remove any doubt, especially when dealing with highly irregular, porous or conductive particles. Appreciable differences have been reported for fly ash particles [6]. This method requires, however, great accuracy in determining the particle concentration in the suspension and a counting efficiency of $100 \%$, i. e. it is necessary to ensure that all particles are counted in order to obtain a precise value for the measured volume.

The experiments were carried out in a Coulter Counter Model $\mathrm{ZM}$, which is claimed to be suitable for accurate analysis. The time needed for the mass calibration of this instrument is considerable. The main feature of this paper concerned the equations which are used to calculate the mass calibration constant. Expressions that involve variables which are not well defined are unreliable. An expression particularly suitable for use with Coulter Counter Model ZM has been derived and validated.

\section{Acknowledgements}

The authors thank Professor B. Scarlett and Dr. H. Merkus (Delft University of Technology) for many helpful discussions.

\section{Symbols and Abbreviations}

$A$

$d$

$\bar{d}$

G

$I$

$K_{\mathrm{d}}$

$K_{\mathrm{do}}$

$\Delta n$

$T$

$\bar{T}$

$T_{\mathrm{L}}$

$\bar{V}_{\mathrm{app}}$

$V_{\text {m }}$

$V_{\mathrm{p}}$
$V_{\mathrm{s}}$

$V_{\mathrm{s}}^{\mathrm{p}}$

$w$

$\rho_{\mathrm{s}}$ attenuation setting

particle diameter

mean diameter (based on the mean volume) for a

particular size interval

amplifier gain setting

aperture current

calibration constant (based on the diameter)

initial estimate of the calibration constant

number of particles in a size interval

threshold value

average threshold (corresponding to $\bar{d}$ )

lower threshold level (Eq. (7))

arbitrary volume (Eq. (6))

apparent volume of particles (Eq. (8))

volume of suspension metered for each count (manometer volume)

volume of particles in a metered volume of suspension total volume of suspension total weight of particles

density of particles

\section{References}

[1] T. Allen: Particle Size Measurement. Chapman and Hall, London 1990.

[2] British Standards Institution: British Standard Methods for Determination of Particle Size of Powders. BS 3406: Part 5: 1983.

[3] C. M. L. Atkinson, R. Wilson: The Mass Integration Method for the Calibration of the Electrical Sensing Zone Technique used for the Sizing and Counting of Fine Particles, in N. G. Stanley-Wood, T. Allen (eds.): Proc. Particle Size Analysis 1981. Wiley Heyden, Chichester 1982.

[4] Coulter Electronics Ltd: Instruction Manual for the Coulter Counter Model ZM, Issue H 1985.

[5] M. P. Cowan, J. G. Harfield: The Linearity and Response of Focussed Apertures. Proc. 4th Europ. Symp. Particle Characterization - PARTEC, Nürnberg 1989.

[6] J. G. Bernard, H. L. Jansma, H. G. Merkus, B. Scarlett: Mass Calibration of PFBC Ash Separated by Density with the Coulter Multisizer: Presentation of Cyclone Efficiency in Three Dimensions, in P. J. Lloyd (ed.): Proc. Particle Size Analysis 1988. Wiley, Chichester 1988. 